Pacific Journal of Mathematics

ON THE IRREDUCIBILITY OF AN INDUCED 


\title{
ON THE IRREDUCIBILITY OF AN INDUCED REPRESENTATION
}

\author{
JoHN C. QUIGG
}

\begin{abstract}
A unitary representation induced from a normal subgroup of a second countable locally compact group with abelian quotient is irreducible if and only if (i) the inducing representation is irreducible with trivial stability subgroup and (ii) the restriction of the induced representation to the normal subgroup is type $I$. This is proved in the context of twisted group algebras using a duality result for induced representations which includes the Takesaki duality theorem for crossed products of von Neumann algebras (having separable pre-dual). Examples are given showing that condition (ii) above is not redundant.
\end{abstract}

1. Introduction. Let $K$ be a second countable locally compact group with closed normal subgroup $N$ and quotient group $G$, and let $\pi$ be a weakly continuous unitary representation of $N$. Denote the induced representation by ind $\pi$ and its restriction to $N$ by $\tilde{\pi}$. When is ind $\pi$ irreducible? It has long been known that the condition

$\pi$ is irreducible with trivial stability subgroup (in $G$ )

is necessary (see, e.g., Mackey [35] and Blattner [2]). It has also been known that (1.1) coupled with the condition

$$
\tilde{\pi} \text { is type } I
$$

is sufficient, for in the presence of (1.1) the condition (1.2) is equivalent with the measure class $\mathscr{C}(\pi, G)$ in $\hat{N}$ (the set of unitary equivalence classes of irreducible representations of $N$ equipped with the Mackey Borel structure) associated with the direct integral decomposition of $\tilde{\pi}$ over $G$ being canonical (Mackey [33]), which is in turn equivalent with the canonical system of imprimitivity based on $G$ being contained in the von Neumann algebra $\mathscr{A}_{\tilde{\pi}}$ generated by $\tilde{\pi}$. Of course, ind $\pi$ is irreducible if and only if (1.1) holds and $\mathscr{R}$ ind $\pi$ contains the system of imprimitivity, but the precise role played by (1.2) with regard to this containment has been unclear up to now. $\mathscr{C}(\pi, G)$ is always canonical if $G$ is discrete (for then the direct integral reduces to a direct sum), or if $N$ is type I, or, more generally, if the kernels of the translates of $\pi$ by $G$ are all distinct (for then the system of imprimitivity can be based on the primitive ideal space of $N$ and the results of Effros [15] applied; see also Blattner [2]). However, it has been conjectured (Mackey [35], Busby 
and Smith [5]) that noncanonical measure classes can in fact occur in the form $\mathscr{C}(\pi, G)$ and that the resulting induced representations must be reducible (because it would then be difficult to imagine $\mathscr{A}_{\text {ind } \pi}$ containing the system of imprimitivity).

In this paper we verify this conjecture when the quotient group is abelian, thus showing that in this case $(1.1,2)$ are necessary and sufficient for the induced representation to be irreducible, and that (1.2) is not redundant. Specifically, the main result of $\S 4$ (Theorem 4.3) shows that (1.2) is a necessary condition, and in $\S 5$ we give examples where (1.1) obtains but (1.2) does not.

We actually work in the more general context of twisted group algebras as developed in Busby and Smith [5] (see also Leptin [26, $27,28,29,30])$. If $N$ is replaced by its group algebra $A=L^{1}(N)$ we can form a twisted covariant system consisting of an automorphic action of $G$ on $A$ which is twisted by a 2-cocycle (the action being unique up to the choice of cocycle), and the resulting twisted group algebra (see $\S 2$ for the precise definition) is naturally isomorphic with $L^{1}(K)$. An induced representation can be defined in terms of the twisted group algebra which is equivalent to the induced representation of $K$ modulo the aforementioned isomorphism.

The theory of twisted covariant systems, twisted group algebras, and their representations only requires $A$ to be a separable Banach *-algebra with bounded approximate identity, and is hence much more general than group extensions, encompassing transformation groups and dynamical systems, covariant systems of mathematical physics, and projective group algebras. In $\S 2$ we list the basic definitions and results of this theory, essentially following Busby and Smith [5]. Related constructions of about the same generality are given in Fell [18], Leinert [24], and Leptin [26, 27, 28, 29, 30], and strong interrelationships between these theories have been found by Busby [4] and Leinert [25]. Other constructions not quite so general can be found in Dang-Ngoc [10] and Green [20], where the group generated by the cocycle is assumed locally compact, and Zeller-Meier [50], where $G$ is discrete, and analogues with no cocycle have been defined by Doplicher, Kastler, and Robinson [14], Effros and Hahn [17], Glimm [19], and Segal [41]. We mention that the constructions of Dang-Ngoc and Green are sufficiently general to include all the applications mentioned above.

In this same section we also give a construction of twisted crossed products of von Neumann algebras which generalizes that of crossed products as defined by Takesaki [49], and we show a close relationship with induced representations, especially in the absence of a cocycle. Choda [7, 8], Rousseau [40], and Sutherland $[43,44]$ have given constructions of essentially the same generality (Rousseau has 
no separability restrictions).

In $\S 3$ we prove a duality theorem for induced representations in the case of $G$ abelian which is very close to that of N. Pedersen [38], although our proof is distinct from his. An almost immediate corollary is the Takesaki duality theorem for crossed products of von Neumann algebras (Takesaki [49]) in the separable pre-dual case. Our technique is heavily representation-theoretic and involves a dual covariant system which is essentially that of Takai [45] (he does not consider a cocycle); see also Green [20, 21].

In $\S 4$ we prove the main result and give several corollaries, among them a result concerning ergodic automorphism groups of von Neumann algebras and maximal abelian subalgebras. The proof of the main result is actually quite short, relying heavily upon the duality theory of $\S 3$ and a simple lemma (Corollary 4.2) concerning certain twisted crossed products of the algebra of bounded operators on a Hilbert space.

Our examples (of irreducible representations having trivial stability subgroups but inducing reducible representations) are presented in $\S 5$. The context is that of twisted group algebras, but the examples could easily be rephrased in terms of group extensions. We remark that our examples disprove Lemma 3.3 (i) of Takai [46]. Takai references the proof of Theorem 4.2 of Busby and Smith [5]. Unfortunately, while this theorem of Busby and Smith is correct, their proof is not. We also present an interesting connection with strongly ergodic group actions on von Neumann algebras, in particular giving new examples of such.

We emphasize our separability assumptions, which remain in force throughout.

2. Twisted group algebras and twisted crossed products.

A twisted covariant system is a quadruple $(G, A ; \tau, \alpha)$ consisting of a separable locally compact group $G$, a separable Banach *-algebra $A$ having a bounded approximate identity, and maps $\tau: G \rightarrow$ Aut $A$ (the isometric *automorphisms of $A$ ) and $\alpha: G \times G \rightarrow \mathscr{C}(A)$ (the unitary double centralizers of $A$ ) satisfying:

$$
\begin{gathered}
\tau_{e}=\iota_{A} \\
\alpha(s, e)=\alpha(e, s)=1 \\
\tau_{s} \tau_{t}=\tau_{s t} A d_{\alpha(s, t)} \\
\alpha(r s, t) \tau_{t}^{-1}(\alpha(r, s))=\alpha(r, s t) \alpha(s, t) \\
\tau \text { is strongly Borel }
\end{gathered}
$$




$$
\alpha \text { is strictly Borel . }
$$

(2.1-6) in particular guarantee that the corresponding group extension $\mathscr{G}$ of $\mathscr{C}(A)$ by $G$ can be made into a (Polish) topological group (Brown [3]). When the cocycle $\alpha$ is absent we say the system is split, and in this case the action $\tau$ is strongly continuous by an old Polish group result of Banach [1].

The twisted group algebra of $(G, A ; \tau, \alpha)$ is the space $L_{\tau, \alpha}^{1}(G, A)$ of Bochner integrable functions from $G$ to $A$ equipped with the structure

$$
\begin{aligned}
\|f\| & =\int_{G}\|f(s)\| d s \\
f^{*}(s) & =\Delta_{G}(s)^{-1} \tau_{s}^{-1}\left(f\left(s^{-1}\right)\right)^{*} \alpha\left(s^{-1}, s\right)^{*} \\
f * g(s) & =\int_{G} \alpha\left(t, t^{-1} s\right) \tau_{t}^{-1_{s}}(f(t)) g\left(t^{-1} s\right) d t,
\end{aligned}
$$

which makes it a separable Banach *-algebra with bounded approximate identity.

$G$ and $M(A)$ (the double centralizers of $A$ ) are embedded in $M\left(L_{\tau, \alpha}^{1}(G, A)\right)$ by

$$
\begin{aligned}
& (s * f)(t)=\alpha\left(s, s^{-1} t\right) f\left(s^{-1} t\right) \\
& (f * s)(t)=\Delta_{G}(s)^{-1} \alpha\left(t s^{-1}, s\right) \tau_{s}^{-1}\left(f\left(t s^{-1}\right)\right) \\
& (x * f)(t)=\tau_{t}^{-1}(x) f(t) \\
& (f * x)(t)=f(t) x
\end{aligned}
$$

and the resulting embedding of $\mathscr{G}$ is a homeomorphic monomorphism.

A representation of $(G, A ; \tau, \alpha)$ in the separable Hilbert space $\mathscr{H}$ will refer either to a non-degenerate *-representation $\Pi$ of $L_{\tau, \alpha}^{1}(G, A)$ in $\mathscr{C}$ or to a pair $(U, \pi)$ consisting of a weakly Borel map $U$ from $G$ to the unitary group $\mathscr{C}(\mathscr{H})$ of $\mathscr{H}$ and a nondegenerate *-homomorphism $\pi$ of $A$ into the bounded operators $\mathscr{L}(\mathscr{H})$ of $\mathscr{L}$, satisfying

$$
\begin{aligned}
& U(s) U(t)=U(s t) \pi \circ \alpha(s, t) \\
& A d_{U(s)} \circ \pi=\pi \circ \tau_{s} .
\end{aligned}
$$

$\Pi$ and $(U, \pi)$ are called the integrated form and the covariant form of the representation, respectively, and they are put into a bijective correspondence by the relationship

$$
\Pi(f)=\int_{G} U(s) \pi(f(s)) d s .
$$

The covariant form of $\Pi$ is recovered by extending $\Pi$ to $M\left(L_{\tau, \alpha}^{1}(G, A)\right)$ and restricting to $\mathscr{G}$. This correspondence respects equivalence, 
quasi-equivalence, and commuting algebras, and we blur the distinction between $\Pi$ and $(U, \pi)$ just as for group representations.

Note that $A d_{U}$ restricts to give an action of $G$ on $\mathscr{A}_{\pi}^{\prime}$ which is split and hence $\sigma$-weakly continuous. Moreover,

$$
\mathscr{A}_{U, \pi}^{\prime}=\left(\mathscr{A}_{\pi}^{\prime}\right)^{G} \text {, }
$$

the fixed-point subalgebra of $\mathscr{A}_{\pi}^{\prime}$ under this action, so that in particular $(U, \pi)$ is irreducible if and only if $G$ acts ergodically on $\mathscr{A}_{\pi}^{\prime}$.

For the representation $\pi$ of $A$ in $\mathscr{H}$ the induced representation ind $\pi=\left(U_{\pi}, \tilde{\pi}\right)$ of $(G, A ; \tau, \alpha)$ in $L^{2}(G, \mathscr{H})$ is defined by

$$
\begin{aligned}
\left(U_{\pi}(s) \xi\right)(t) & =\pi \circ \alpha\left(s, s^{-1} t\right) \xi\left(s^{-1} t\right) \\
(\tilde{\pi}(x) \xi)(t) & =\pi \circ \tau_{t}^{-1}(x) \xi(t) .
\end{aligned}
$$

The translate of $\pi$ by $s$ is defined as $s \cdot \pi=\pi \circ \tau_{s}^{-1}$, so that

$$
\tilde{\pi}=\int_{G}^{\oplus} s \cdot \pi d s .
$$

The integrated form of ind $\pi$ is given by

$$
(\text { ind } \pi(f) \xi)(t)=\int_{G} \pi \circ \alpha\left(s, s^{-1} t\right) s^{-1} t \cdot \pi(f(s)) \xi\left(s^{-1} t\right) d s .
$$

When clarity is endangered we write $\operatorname{ind}_{G} \pi$.

The fundamental properties of induced representations, the proofs of which are either straightforward or may be found in, e.g., Busby and Smith [5], are collected in the following proposition:

\section{Proposition 2.1.}

(i) ind $\pi \cong$ ind $s \cdot \pi(s \in G)$.

(ii) $\pi_{1} \cong($ resp. $\sim) \pi_{2} \Rightarrow$ ind $\pi_{1} \cong($ resp. $\sim)$ ind $\pi_{2}$.

(iii) $G$ acts in $\hat{A}$ as a transformation group by $s \cdot[\pi]=[s \cdot \pi]$, and [ind $\pi$ ] depends only upon the orbit $G \cdot[\pi]$.

(iv) If $s \rightarrow \pi_{s}$ is a Borel field of representations of $A$ based on the standard measure space $(S, \mu)$, then

$$
\text { ind } \int_{S}^{\oplus} \pi_{s} d \mu(s) \cong \int_{S}^{\oplus} \text { ind } \pi_{s} d \mu(s) .
$$

(v) ind $\pi \sim$ ind $\tilde{\pi}$.

(vi) $\mathscr{A}_{\text {ind } \pi}^{\prime} \cap\left(L^{\infty}(G) \otimes 1\right)^{\prime}=1_{L^{2}(G)} \otimes \mathscr{A}_{\pi}^{\prime}$.

$L^{\infty}(G) \otimes 1$ is the canonical system of imprimitivity for ind $\pi$, and Proposition 2.1(vi) shows that the von Neumann algebra generated by ind $\pi$ and its canonical system of imprimitivity is $\mathscr{L}\left(L^{2}(G)\right) \otimes \mathscr{A} \pi$.

If $\mathscr{A}$ is a von Neumann algebra (concretely represented on a 
separable Hilbert space $\mathscr{H}$ ) with separable pre-dual, a twisted $W^{*}$ covariant system $(G, \mathscr{A} ; \theta, \beta)$ is defined in a manner exactly analogous to twisted covariant systems, except that the topologies are taken as is appropriate for a von Neumann algebra. Of course, $M(\mathscr{A})=\mathscr{A}$ since $\mathscr{A}$ has an identity, and instead of the strict topology on $\mathscr{U}(\mathscr{A})$ we use the weak topology.

The twisted crossed product of $G$ and $\mathscr{A}$ is the von Neumann algebra $\mathscr{L}_{\theta, \beta}(G, \mathscr{A})$ on $L^{2}(G, \mathscr{H})$ generated by the operators $\left\{U_{\theta}(s)\right.$, $\left.\pi_{\theta}(x) \mid s \in G, x \in \mathscr{A}\right\}$, where

$$
\begin{aligned}
& \left(U_{\theta}(s) \xi\right)(t)=\beta\left(s, s^{-1} t\right) \xi\left(s^{-1} t\right) \\
& \left(\pi_{\theta}(x) \xi\right)(t)=\theta_{t}^{-1}(x) \xi(t) .
\end{aligned}
$$

If the system is split this reduces to the usual crossed product, since $\theta$ is then automatically continuous.

It is possible (see, e.g., Quigg [39]) to develop a representation theory for $(G, \mathscr{A} ; \theta, \beta)$ paralleling that for $(G, A ; \tau, \alpha)$, including a convolution algebra of $\mathscr{A}$-valued functions, and in this context $\mathscr{L}_{\theta, \beta}(G, \mathscr{A})$ is the von Neumann algebra generated by the representation induced from the identity representation of $\mathscr{A}$. In particular, one of the fundamental properties of induced representations guarantees that the isomorphism class of the twisted crossed product is independent of the choice of realization of $\mathscr{A}$.

Let $(U, \pi)$ be a representation of the twisted covariant system $(G, A ; \tau, \alpha)$. Then it is almost obvious that $\left(G, \mathscr{A}_{\pi} ; A d_{U}, \pi \circ \alpha\right)$ is a twisted $W^{*}$-covariant system (observe that $\pi: \mathscr{U}(A) \rightarrow \mathscr{U}(\mathscr{H})$ is continuous from the strict topology to the weak topology) and that

$$
\mathscr{A}_{\text {ind } \pi}=\mathscr{L}_{A_{U}, \pi \circ \alpha}\left(G, \mathscr{A}_{\pi}\right) \text {. }
$$

The following proposition shows a partial converse, namely that every (split) $W^{*}$-covariant system is generated by a (split) covariant system, and therefore that every crossed product arises as the von Neumann algebra generated by an induced representation of a covariant system. The proof, modulo a minor modification to take care of the separability requirements, can be found in Takesaki [49].

Proposition 2.2. If $(G, \mathscr{A} ; \theta)$ is a $W^{*}$-covariant system, then there exists a separable G-invariant $\sigma$-weakly dense $C^{*}$-subalgebra of $\mathscr{A}$ on which the action is strongly continuous.

3. Dual covariant systems. Throughout this section $(G, A ; \tau, \alpha)$ will be a twisted covariant system with $G$ abelian. For $\chi \in \hat{G}$ (the dual group of $G)$ and $f \in L_{\tau, \alpha}^{1}(G, A)$ define $\hat{\tau}_{\chi}(f) \in L_{\tau, \alpha}^{1}(G, A)$ by

$$
\hat{\tau}_{\chi}(f)(s)=\overline{\langle s, \chi\rangle} f(s) \text {. }
$$


Proposition 3.1. $\hat{\tau}$ is a strongly continuous action of $\hat{G}$ on $L_{\tau, \alpha}^{1}(G, A)$.

Proof. A routine computation shows that $\hat{\tau}$ is a homomorphism from $\hat{G}$ to Aut $L_{\tau, \alpha}^{1}(G, A)$, and the continuity follows from the Lebesgue dominated convergence theorem.

We call $\hat{\tau}$ the dual action and $\left(\widehat{G}, L_{\tau, \alpha}^{1}(G, A) ; \hat{\tau}\right)$ the dual covariant system.

Lemma 3.2. If $\Pi=(U, \pi)$ is a representation of $(G, A ; \tau, \alpha)$, then

$$
\chi \cdot \Pi=(\chi U, \pi)
$$

Proof.

$$
\begin{aligned}
\chi \cdot \Pi(f) & =\Pi \circ \hat{\tau}_{\chi}^{-1}(f) \\
& =\int_{G} U(s) \pi\left(\hat{\tau}_{\chi}^{-1}(f)(s)\right) d s \\
& =\int_{G}\langle s, \chi\rangle U(s) \pi(f(s)) d s \\
& =(\chi U, \pi)(f) .
\end{aligned}
$$

We use this formula to show that an induced representation of $(G, A ; \tau, \alpha)$ can be extended to $\left(\hat{G}, L_{\tau, \alpha}^{1}(G, A) ; \hat{\tau}\right)$. Let $\pi$ be a representation of $A$ in $\mathscr{H}$, and define the representation $\hat{U}_{\pi}$ of $\hat{G}$ in $L^{2}(G, \mathscr{H})$ by

$$
\left(\hat{U}_{\pi}(\chi) \xi\right)(s)=\overline{\langle s, \chi\rangle} \xi(s)
$$

Proposition 3.3. $\hat{\Pi}=\left(\hat{U}_{\pi}\right.$, ind $\left.\pi\right)$ is a representation of $(\hat{G}$, $\left.L_{\tau, \alpha}^{1}(G, A) ; \hat{\tau}\right)$.

Proof. We must show

$$
A d_{\hat{U}_{\pi}(x)} \circ \text { ind } \pi=(\text { ind } \pi) \circ \widehat{\tau}_{x} .
$$

Clearly $\hat{U}_{\pi}(\chi)$ commutes with $\tilde{\pi}$, and

$$
\begin{aligned}
\left(A d_{\hat{U}_{\pi}(x)}\left(U_{\pi}(s)\right) \xi\right)(t) & =\overline{\langle t, \chi\rangle} \pi \circ \alpha\left(s, s^{-1} t\right)\left\langle s^{-1} t, \chi\right\rangle \xi\left(s^{-1} t\right) \\
& =\left(\overline{\langle s, \chi\rangle} U_{\pi}(s) \xi\right)(t),
\end{aligned}
$$

so we have

$$
A d_{\hat{U}_{\pi}(x)} \circ \text { ind } \pi=\left(\chi^{-1} U_{\pi}, \tilde{\pi}\right),
$$

and this last representation is (ind $\pi$ ) $\circ \hat{\tau}_{x}$ by Lemma 3.2. 
We call $\hat{\Pi}$ the dual representation. Now assume that $\pi$ extends to a representation $\Pi=(U, \pi)$ of $(G, A ; \tau, \alpha)$. It turns out that in this situation the dual representation is induced. Define the unitary $W: L^{2}(G, \mathscr{H}) \rightarrow L^{2}(\hat{G}, \mathscr{H})$ by

$$
(W \xi)(\chi)=\int_{G}\langle s, \chi\rangle U(s) \xi(s) d s .
$$

Proposition 3.4. W implements an equivalence between $\hat{\Pi}$ and $\operatorname{ind}_{\hat{G}} \Pi$.

Proof. First of all we have

$$
\begin{aligned}
\left(W \hat{U}_{\pi}(\chi) \xi\right)(\gamma) & =\int_{G}\langle s, \gamma\rangle U(s)\left(\hat{U}_{\pi}(\chi) \xi\right)(s) d s \\
& =\int_{G}\langle s, \gamma\rangle U(s) \overline{\langle s, \chi\rangle} \xi(s) d s \\
& =\int_{G}\left\langle s, \chi^{-1} \gamma\right\rangle U(s) \xi(s) d s \\
& =(W \xi)\left(\chi^{-1} \gamma\right) \\
& =\left(U_{I I}(\chi) W \xi\right)(\gamma)
\end{aligned}
$$

Next,

$$
\begin{aligned}
\left(W U_{\pi}(s) \xi\right)(\gamma) & =\int_{G}\langle t, \gamma\rangle U(t)\left(U_{\pi}(s) \xi\right)(t) d t \\
& =\int_{G}\langle t, \gamma\rangle U(t) \pi \circ \alpha\left(s, s^{-1} t\right) \xi\left(s^{-1} t\right) d t \\
& =\int_{G}\langle s t, \gamma\rangle U(s t) \pi \circ \alpha(s, t) \xi(t) d t \\
& =\langle s, \gamma\rangle U(s) \int_{G}\langle t, \gamma\rangle U(t) \xi(t) d t \\
& =\langle s, \gamma\rangle U(s)(W \xi)(\gamma),
\end{aligned}
$$

and

$$
\begin{aligned}
(W \tilde{\pi}(x) \xi)(\gamma) & =\int_{G}\langle s, \gamma\rangle U(s)(\tilde{\pi}(x) \xi)(s) d s \\
& =\int_{G}\langle s, \gamma\rangle U(s) s \cdot \pi(x) \xi(s) d s \\
& =\int_{G}\langle s, \gamma\rangle \pi(x) U(s) \xi(s) d s \\
& =\pi(x)(W \xi)(\gamma) .
\end{aligned}
$$

Thus

$$
\begin{aligned}
A d_{W} \circ \operatorname{ind}_{G} \pi & =\left(\int_{\hat{G}}^{\oplus} \chi U d \chi, 1_{L^{2}(G)} \otimes \pi\right) \\
& =\int_{\hat{G}}^{\oplus}(\chi U, \pi) d \chi
\end{aligned}
$$




$$
\begin{aligned}
& =\int_{\widehat{G}}^{\oplus} \chi \cdot \Pi d \chi \\
& =\widetilde{\Pi} .
\end{aligned}
$$

We are now in a position to prove a duality theorem for induced representations. Call a representation of a $A$ quasi-invariant if its translates are all quasi-equivalent.

THEOREM 3.5. If $\pi$ is a quasi-invariant representation of $A$, then

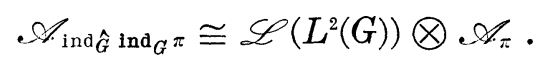

Proof. Without loss of generality we may assume $\pi$ extends to a representation $\Pi$ of $(G, A ; \tau, \alpha)$, since $\tilde{\pi}$ does and the quasi-invariance of $\pi$ guarantees that $\pi \sim \tilde{\pi}$. Then

$$
\begin{aligned}
\operatorname{ind}_{\hat{G}} \operatorname{ind}_{G} \pi & \cong \operatorname{ind}_{\hat{G}} \widetilde{\Pi} \\
& \sim \operatorname{ind}_{\hat{G}} \Pi \\
& \cong \widetilde{\Pi},
\end{aligned}
$$

where we have applied Proposition 3.4 twice.

Now, $\widehat{G}$ is $\sigma$-weakly total in $L^{\infty}(G)$, so $\widehat{U}_{\pi}$ generates the canonical system of imprimitivity for $\operatorname{ind}_{G} \pi$. Thus we have

$$
\begin{aligned}
& \mathscr{\mathscr { A }}_{\operatorname{ind}_{\hat{G}} \operatorname{ind}_{G} \pi} \cong \mathscr{A}_{\hat{\Pi}} \\
&=\mathscr{L}\left(L^{2}(G)\right) \otimes \mathscr{\mathscr { A } _ { \pi }} .
\end{aligned}
$$

Our first corollary is the separable case of the Takesaki duality theorem for crossed products (Takesaki [49]).

Corollary 3.6. If $(G, \mathscr{A} ; \theta)$ is a $W^{*}$-covariant system with $G$ abelian, then there is an action $\hat{\theta}$ of $\hat{G}$ on $\mathscr{L}_{\theta}(G, \mathscr{A})$ such that

$$
\mathscr{L}_{\hat{\theta}}\left(\hat{G}, \mathscr{L}_{\theta}(G, \mathscr{A})\right) \cong \mathscr{L}\left(L^{2}(G)\right) \otimes \mathscr{A} \text {. }
$$

Proof. Since the system is split, there is a $\sigma$-weakly dense $C^{*}$-subalgebra of $\mathscr{A}$ on which the action is strongly continuous (Proposition 2.2), and this allows us to use Theorem 3.5, keeping in mind the connection between crossed products and induced representations (§2).

The form in which we will use Theorem 3.5 is expressed in the

COROLlaRY 3.7. If $\pi$ is a representation of $A$, then 


$$
\mathscr{A}_{\operatorname{ind}_{\hat{G}} \operatorname{ind}_{G} \pi} \cong \mathscr{L}\left(L^{2}(G)\right) \otimes \mathscr{L}_{\tilde{\pi}}
$$

Proof. This follows immediately from Theorem 3.5 since $\operatorname{ind}_{G} \pi \sim$ $\operatorname{ind}_{G} \tilde{\pi}$ and $\tilde{\pi}$ is quasi-invariant.

4. Irreducibility of induced representations. We begin with a straightforward generalization (to account for the cocycle) of Lemma 2.1 of Landstad [23]. We only need the special case stated in the corollary.

We say that the twisted $W^{*}$-covariant system $(G, \mathscr{A} ; \theta, \beta)$ is implemented by the weakly Borel map $U: G \rightarrow \mathscr{U}(\mathscr{C})$ if $\theta=A d_{U}$ and $U$ satisfies

$$
U(s) U(t)=U(s, t) \beta(s, t) .
$$

Let $\mathscr{L}(G)$ denote the von Neumann algebra generated by the left regular representation of $G$.

LEMMA 4.1. If $(G, \mathscr{A} ; \theta, \beta)$ is a twisted $W^{*}$-covariant system which is implemented by $U$ and $U(G) \subset \mathscr{A}$, then

$$
\mathscr{L}_{\theta, \beta}(G, \mathscr{A}) \cong \mathscr{L}(G) \otimes \mathscr{A}
$$

CoROLlaRy 4.2. Let $(G, A ; \tau, \alpha)$ be a twisted covariant system with $G$ abelian, and let $\pi$ be an irreducible representation of $A$. If $\pi$ extends to $(G, A ; \tau, \alpha)$, then ind $\pi$ is type $\mathrm{I}$.

Proof. This follows immediately from Lemma 4.1 and the connection between twisted crossed products and induced representations (§ 2).

We remark that in the context of a locally compact group extension with abelian quotient this says that if an irreducible representation of the normal subgroup extends to the whole group, then the induced representation is type $I$.

We are now prepared for the main result:

THEOREM 4.3. Let $(G, A ; \tau, \alpha)$ be a twisted covariant system with $G$ abelian, and let $\pi$ be a representation of $A$. Then ind $\pi$ is irreducible if and only if $\pi$ is irreducible with trivial stability subgroup and $\tilde{\pi}$ is type I.

Proof. As we have pointed out in the introduction, we need only prove the necessity of the condition concerning $\tilde{\pi}$. By the results of $\S 3$, there is a dual action $\hat{\tau}$ of $\hat{G}$ on $L_{\tau, \alpha}^{1}(G, A)$ such that 


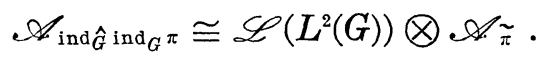

Thus $\tilde{\pi}$ must be type I if $\operatorname{ind}_{\hat{G}} \operatorname{ind}_{G} \pi$ is. Section 3 also gives us an extension of $\operatorname{ind}_{G} \pi$ to $\left(\hat{G}, L_{\tau, \alpha}^{1}(G, A) ; \hat{\tau}\right)$. Thus, if ind $\pi$ is irreducible, Corollary 4.2 assures us that $\operatorname{ind}_{\hat{G}} \operatorname{ind}_{G} \pi$ is indeed type I.

We present three corollaries, the first being a reformulation in terms of the Davies Borel structure (Davies [11]), and the others concerning ergodic actions on von Neumann algebras and maximal abelian subalgebras.

COROLlaRy 4.4. With the hypotheses of Theorem 4.3, if $\pi$ is irreducible with trivial stability subgroup, then the following are equivalent:

(i) ind $\pi$ is irreducible.

(ii) The map $s \in G \rightarrow s \cdot[\pi] \in \widehat{A}$ induces the relative Davies Borel structure on a co-null Borel subset of the orbit $G \cdot[\pi]$.

(iii) There is a sequence $\left\{x_{j}\right\}$ in $\mathscr{F}(M(A)$ ) (the center of $M(A)$ ) having the property that for all $s \neq e$ there is a $j$ such that

$$
s \cdot \pi\left(x_{j}\right) \neq \pi\left(x_{j}\right) .
$$

Proof. Effros [16] has shown that a measure class in $\hat{A}$ is canonical if and only if there is a co-null Borel set on which the Davies and Mackey Borel structures agree and are standard, and furthermore if and only if there is a co-null set $S$ in $\hat{A}$ and countably many elements of $\mathscr{Z}(M(A))$ which separate the points of $S$ (recall that for $x \in \mathscr{Z}(M(A))$ and $\pi$ a factor representation of $A$ the operator $\pi(x)$ is a multiple of the identity, this multiple only depending upon the quasi-equivalence class of $\pi$ ). Since $G$ acts as a transformation group in $\hat{A}$ the set $\left\{x_{j}\right\}$ will separate $G \cdot[\pi]$ if and only if it separates $(\mathrm{G}-\{e\}) \cdot[\pi]$ from $[\pi]$.

COROLlaRY 4.5. Let the abelian group $G$ act (without cocycle) on the von Neumann algebra $\mathscr{A}$, restricting to a free and transitive action on an abelian von Neumann subalgebra $\mathscr{L}$. Then the action is ergodic on $\mathscr{A}$ if and only if $\mathscr{A}$ is type I and $\mathscr{C l}$ is maximal abelian and simple (in the sense of Takesaki [47]).

Proof. Assume without loss of generality that the action is implemented by the representation $U$ of $G$ (e.g., in the crossed product representation). Then $G$ also acts by $A d_{U}$ on $\mathscr{A}^{\prime}$, and there exists a separable $\sigma$-weakly dense $C^{*}$-subalgebra $A$ of $\mathscr{C}^{\prime}$ on which this action is strongly continuous (Proposition 2.2). Then $\left(U, \iota_{A}\right)$ is a representation of the covariant system $\left(G, A ; A d_{U}\right)$ with a free and 
transitive system of imprimitivity $\mathscr{C}$, so there is a representation $\pi$ of $A$ such that

$$
\left(U, \iota_{A}\right) \cong\left(U_{\pi}, \tilde{\pi}\right)=\text { ind } \pi
$$

(Takesaki [48]). Thus, $G$ acts ergodically on $\mathscr{A}$ if and only if ind $\pi$ is irreducible. By Theorem 4.3 this happens if and only if $\tilde{\pi}$ is type I and $\pi$ is irreducible with trivial stability subgroup, i.e., if and only if $\mathscr{A}$ is type I and $\mathscr{C}$ is maximal abelian and simple in $\mathscr{A} . \square$

COROLLARY 4.6. If a discrete abelian group acts (without cocycle) on the von Neumann algebra $\mathscr{A}$, restricting to a free and transitive action on a simple maximal abelian subalgebra $\mathscr{L}$, then $\mathscr{A}$ is type I and the action is ergodic on .A.

Proof. In the notation of the above proof, we have $\pi$ irreducible with trivial stability subgroup. $G$ being discrete, we must have $\tilde{\pi}$ type $I$ and ind $\pi$ irreducible.

5. Examples, Let $K$ be a separable locally compact group which is a semi-direct product of a closed normal subgroup $N$ and its abelian quotient group $H$, and denote by $\sigma$ the corresponding action of $H$ on $N$. Then we can form a covariant system $\left(H, L^{1}(N) ; \kappa\right)$ such that $L_{\kappa}^{1}\left(H, L^{1}(N)\right)$ is naturally isomorphic with $L^{1}(K)$. Let $A=$ $L^{1}\left(H, L^{1}(N)\right), G=\hat{H}$, and $\tau=\hat{\kappa}$, so that $(G, A ; \tau)$ is the dual covariant system to $\left(H, L^{1}(N) ; \kappa\right)$. Let $\lambda_{N}$ be the left regular representation of $N$, and define the representation $V_{\sigma}$ of $H$ in $L^{2}(N)$ by

$$
V_{o}(h) \xi=\Delta_{\sigma}(h)^{1 / 2} \xi \circ \sigma_{h}^{-1},
$$

where $\Delta_{\sigma}$ is the modular function of the action $\sigma$. Then $\pi=\left(V_{o}, \lambda_{N}\right)$ is a representation of $A$; indeed, blurring the distinction between $A$ and $L^{1}(K)$,

$$
\pi \cong \operatorname{ind}_{H \uparrow K} 1_{H},
$$

where $1_{H}$ is the identity character of $H$ and the induction is in the Mackey sense. Moreover, for $\chi \in G$

$$
\chi \cdot \pi \cong \operatorname{ind}_{H \uparrow K} \chi,
$$

and we have

$$
\tilde{\pi} \cong \lambda_{K} .
$$

Thus, in order that $\pi$ be irreducible with trivial stability subgroup, we need $\left\{\operatorname{ind}_{H \uparrow K} \chi \mid \chi \in G\right\}$ to be irreducible and pair-wise inequivalent. If $H$ and $N$ are discrete, this latter condition holds if the $\sigma$-orbit 
of every nonidentity element of $N$ is infinite (Mackey [31, $\S 3]$; he also makes, but does not use, the assumption that $N$ is abelian). Observe that if $\sigma$ is free, i.e., $\sigma_{h}(n) \neq n$ for $h$ and $n$ not the identity, which is a stronger condition (assume $H$ is infinite), then $K$ is ICC (infinite conjugacy class), so that $\tilde{\pi}$ is a $I I_{1}$ factor representation. Also, if $N$ is amenable, then so is $K$, so that $\tilde{\pi}$ and ind $\pi$ are approximately finite-dimensional (see, e.g., Connes [9, introduction]).

The duality theory of $\S 3$ guarantees that ind $\pi$ is equivalent to the dual representation of $\lambda_{N}$, so that

$$
\mathscr{A}_{\text {ind } \pi}^{\prime} \cong \mathscr{R}(N) \text {, }
$$

the right regular von Neumann algebra of $N$.

Thus, in this situation, if $\sigma$ is free, then $\pi$ is irreducible with trivial stability subgroup but ind $\pi$ is reducible and $\tilde{\pi}$ is a $I I_{1}$ factor representation.

EXAMPLE 5.1. Let $H=\boldsymbol{Q}_{+}^{*}$ (the positive rationals under multiplication) and $N=\boldsymbol{Q}$ (the rationals under addition), and define the action $\sigma$ by

$$
\sigma_{h}(n)=h n .
$$

Then $K$ is the rational $a x+b$ group. $\sigma$ is clearly free, and $N$ is abelian, so ind $\pi$ is reducible and multiplicity-free and $\tilde{\pi}$ generates the hyperfinite $I I_{1}$ factor.

Note that if $H=\boldsymbol{Z}$ (the integers) then $\sigma$ is free if and only if $\sigma_{1}$ is not periodic on any nonidentity element, in which case we say $\sigma_{1}$ is aperiodic.

ExAMPLe 5.2. Let $H=Z$ and $N=\Pi_{\infty}$ (the group of finite permutations of $Z$ ), and define the automorphism $\sigma_{1}$ of $N$ by

$$
\sigma_{1}(T)=S T S^{-1},
$$

where $S$ is the shift permutation of $Z$ :

$$
S(n)=n+1 .
$$

Then, denoting the fixed point set of $T \in \Pi_{\infty}$ by $F_{T}$ (which is co-finite), we have $F_{o_{1}(T)}=F_{T}+1$, so $\sigma_{1}$ is aperiodic. Moreover, $N$ is amenable (being an increasing union of finite subgroups), so ind $\pi$ and $\tilde{\pi}$ both generate the hyperfinite $I I_{1}$ factor.

EXAMPLE 5.3. Let $H=Z$ and $N=F_{\infty}$ (the free group on the generators $\left\{a_{n} \mid n \in Z\right\}$ ), and define the automorphism $\sigma_{1}$ of $N$ by shifting the generators: 


$$
\sigma_{1}\left(a_{n}\right)=a_{n+1} .
$$

Then $\sigma_{1}$ is clearly aperiodic, and $N$ (hence $K$ ) is nonamenable, so in this case ind $\pi$ and $\tilde{\pi}$ generate nonhyperfinite $I I_{1}$ factors.

We now generalize slightly in order to obtain an interesting connection with strongly ergodic actions. Recall (Nielsen [36]) that an action of a group on a von Neumann algebra is said to be strongly ergodic if the only common eigenoperators are constant.

Let $(H, B ; \kappa, \beta)$ be a twisted covariant system with $H$ abelian, $(G, A ; \tau)$ the dual covariant system, and $\pi=(V, \lambda)$ a representation of $A$.

Proposition 5.4. (i) ind $\pi$ is irreducible if and only if $\lambda$ is.

(ii) $\pi$ is irreducible with trivial stability subgroup if and only if the action $\theta=A d_{U}$ of $H$ on $\mathscr{A}_{\lambda}^{\prime}$ is strongly ergodic. Moreover, in this case $\pi$ is type I if and only if $\lambda$ is irreducible.

Proof. (i) We see that $\mathscr{A}_{\text {ind } \pi}^{\prime} \cong \mathscr{A}_{2}^{\prime}$ in the same way as in the discussion given for the case $B=L^{1}(N)$.

(ii) Recall that $\pi$ is irreducible if and only if $H$ acts ergodically on $\mathscr{A}_{\lambda}^{\prime}$. Since the translate of $\pi$ by $\chi \in G$ is given by

$$
\chi \cdot \pi=(\chi V, \lambda)
$$

(Lemma 3.2), $\pi$ has disjoint translates if and only if for $\chi \neq 1_{H} \in G$ there is no nonconstant operator $x \in \mathscr{A}_{\lambda}^{\prime}$ such that

$$
\theta_{h}(x)=\langle h, \chi\rangle x
$$

for all $h \in H$. Thus $\pi$ is irreducible with disjoint (hence inequivalent) translates if and only if $\theta$ is strongly ergodic on $\mathscr{A}_{2}^{\prime}$.

The last statement of (ii) follows immediately from (i) and Theorem 4.3.

CoROllaRY 5.5. Let $(H, \mathscr{A} ; \kappa)$ be a $W^{*}$-covariant system with $H$ abelian and $\kappa$ implemented, and let the associated action of $H$ on $\mathscr{A}$ ' be strongly ergodic. Then $\mathscr{L}_{\kappa}(H, \mathscr{A})$ is type I if and only if $\mathscr{A}=\mathscr{L}(\mathscr{H})$.

Proof. Letting $B$ be a separable $\sigma$-weakly dense $C^{*}$-subalgebra of $\mathscr{C}$ on which $\kappa$ is strongly continuous (Proposition 2.2) and $\lambda$ the identity representation of $B$, we see that $\mathscr{L}_{\kappa}(H, \mathscr{A})$ is the von Neumann algebra generated by the representation ind $\lambda$ of the covariant system $(H, B ; \kappa)$. Letting $V$ be a representation of $H$ which implements $\kappa$, we get a representation $\pi=(V, \lambda)$ of the dual 
covariant system $(G, A ; \tau)$. But ind $\lambda \cong \tilde{\pi}$ (Proposition 3.4 ), and $\tilde{\pi}$ is type $I$ if and only if $\lambda$ is irreducible (Proposition 5.4).

COROLLARY 5.6. Every strongly ergodic action of an abelian group on a von Neumann algebra of dimension greater than one gives rise to an example of the desired sort.

Proof. The "desired sort" of example consists of a twisted covariant system $(G, A ; \tau, \alpha)$ and an irreducible representation $\pi$ of $A$ with trivial stability subgroup such that ind $\pi$ is reducible. Let $\left(H, \mathscr{L}^{\prime} ; \theta\right)$ be a $W^{*}$-covariant system with $H$ abelian and $\theta$ strongly ergodic, and assume without loss of generality that $\theta$ is implemented. Then there is an associated (implemented) system $(H, \mathscr{A} ; \kappa)$, and the proof of Corollary 5.5 gives a covariant system $(G, A ; \tau)$ and an irreducible representation $\pi$ of $A$ with trivial stability subgroup such that $\tilde{\pi}$ is not type I (since $\mathscr{A} \neq \mathscr{L}(\mathscr{H})$ ), and so ind $\pi$ is reducible by Theorem 4.3.

The following corollary is a very weak form of Størmer [42, Theorem 3.4], where different techniques are used. See also Paschke [37, Proposition 4.2].

COROLLARY 5.7. A compact abelian group cannot act strongly ergodically on a von Neumann algebra of dimension greater than one.

Proof. In the notation of the above proof, if $H$ were compact, then $G=\hat{H}$ would be discrete, forcing ind $\pi$ to be irreducible, which is a contradiction.

Finally, we remark that the only examples of strongly ergodic actions we have found in the literature have been on abelian von Neumann algebras (Nielsen [36]), whereas our Examples 5.2 and 5.3 give rise to strongly ergodic actions on nontype I von Neumann algebras.

Acknowledgement. This paper is taken mostly from my doctoral dissertation at Drexel University. I welcome this opportunity to express my deep gratitude to Professor Robert C. Busby, my advisor, for his constant encouragement and for invaluable conversations on many subjects.

\section{REFERENCES}

1. S. Banach, Über metrische gruppen, Studia Math., 3 (1931), 101-113. 
2. R. Blattner, Group extension representations and the structure space, Pacific J. Math., 15 (1965), 1101-1113.

3. L. Brown, Extensions of topological groups, Pacific J. Math., 39 (1971), 71-78.

4. R. Busby, On the equivalence of twisted group algebras and Banach*-algebraic bundles, Proc. Amer. Math. Soc., 37 (1973), 142-148.

5. R. Busby and H. Smith, Representations of twisted group algebras, Trans. Amer. Math. Soc., 149 (1970), 503-537.

6. L. Calabi, Sur les extensions des groupes topologiques, Ann. Math. Pure et Appl., 32 (1951), 295-370.

7. M. Choda, Some relations of $I I_{1}$-factors on free groups, Math. Japonica, 22 (1977), 383-394.

8. Extensions of the inner automorphism group of a factor, Publ. RIMS Kyoto Univ., 14 (1978), 731-739.

9. A. Connes, Classification of injective factors, Ann. of Math., 104 (1976), 73-115.

10. N. Dang-Ngoc, Produits croisés restreints et extensions des groupes, preprint.

11. E. Davies, On the Borel structure of $C^{*}$-algebras, Comm. Math. Phys., 8 (1968), 147-163.

12. J. Dixmier, Les algèbres d'opérateurs dans l'espace hilbertien (algèbres de von Neu. mann), 2nd Ed., Gauthier-Villars, Paris, 1969.

13. - C*algebras, North-Holland, Amsterdam, 1977.

14. S. Doplicher, D. Kastler and D. Robinson, Covariance algebras in field theory and statistical mechanics, Comm. Math. Phys., 3 (1966), 1-28.

15. E. Effros, $A$ decomposition theorem for representations of $C^{*}$-algebras, Trans. Amer. Math. Soc., 107 (1963), 83-106.

16. - The canonical measures for a separable $C^{*}$-algebra, Amer. J. Math., 92 (1970), 56-60.

17. E. Effros and F. Hahn, Locally compact transformation groups and $C^{*}$-algebras, Mem. Amer. Math. Soc., 75 (1967).

18. J. Fell, An extension of Mackey's method to Banach*.algebraic bundles, Mem. Amer. Math. Soc., 90 (1969).

19. J. Glimm, Families of induced representations, Pacific J. Math., 12 (1962), 885-911.

20. P. Green, The local structure of twisted covariance algebras, Acta. Math., 140 (1978), 191-250.

21. The structure of imprimitivity algebras, preprint.

22. A. Guichardet, Sur la décomposition des représentations des $C^{*}$-algèbres, C.R. Acad.

Sci. Paris, 258 (1964), 768-770.

23. M. Landstad, Duality theory for covariant systems, Trans. Amer. Math. Soc., 248 (1979), 223-267.

24. M. Leinert, Beitrag zur Theorie der verallgemeinerten $L^{1}$-Algebren, Acta. Math. (Basel), 21 (1970/71), 594-600.

25. - Fell-Bündle und verallgeminerte $L^{1}$-Algebren, J. Functional Analysis, 22 (1976), 323-345.

26. H. Leptin, Verallgemeinerte $L^{1}$-Algebren, Math. Anal., 159 (1965), 51-76.

27. - Verallgemeinerte $L^{1}$-Algebren und projektive Darstellungen lokal kompakter Gruppen, I, Invent. Math., 3 (1967), 257-281.

28. - Verallgemeinerte $L^{1}$-Algebren und projektive Darstellungen lokal kompakter Gruppen, II, Invent. Math., 4 (1967), 68-86.

29. - Darstellungen verallgemeinerter $L^{1}$-Algebren, Invent. Math., 5 (1968), $192-215$.

30. - Darstellungen verallgemeinerter $L^{1}$-Algebren, II, Lectures on Operator Algebras, 251-307, Lecture Notes in Math., No. 247, Springer-Verlag, Berlin, 1972.

31. G. Mackey, On induced representations of groups, Amer. J. Math., 73 (1951), 576-592. 32. - Induced representations for locally compact groups, I, Ann. of Math., 55 (1952), 101-139. 
33. G. Mackey, Borel structures in groups and their duals, Trans. Amer. Math. Soc., 85 (1957), 134-165.

34. - Unitary representations of group extensions, I, Acta. Math., 99 (1958), 265-311.

35. - Induced representations and normal subgroups, Proc. Int. Symp. Linear Spaces, 319-326, Pergamon, Oxford, 1961.

36. O. Nielsen, Maximal abelian subalgebras of hyperfinite factors, II, J. Functional Analysis, 6 (1970), 192-202.

37. W. Paschke, Inner product modules arising from compact automorphism groups of von Neumann algebras, Trans. Amer. Math. Soc., 224 (1976), 87-102.

38. N. Pedersen, Duality for induced representations and induced weights, Univ. of Copenhagen, preprint No. 8, 1978.

39. J. Quigg, On the irreducibility of an induced representation, Dissertation, Drexel University, 1979.

40. R. Rousseau, The covariance algebra of an extended covariant system, Math. Proc. Cambridge Phil. Soc., 85 (1979), 271-280.

41. I. Segal, A class of operator algebras which are determined by groups, Duke Math. J., 18 (1951), 221-265.

42. E. Størmer, Spectra of ergodic transformations, J. Functional Analysis, 15 (1974), 202-215.

43. C. Sutherland, Cohomology and extensions of von Neumann algebras, I, Queen's Math. preprint (1975).

44. C Cohomology and extensions of von Neumann algebras, II, Oslo preprint (1976).

45. H. Takai, On a duality for crossed products of $C^{*}$-algebras, J. Functional Analysis, 19 (1975), 23-29.

46. - The quasi-orbit space of continuous $C^{*}$-dynamical systems, Trans. Amer. Math. Soc., 216 (1976), 105-113.

47. M. Takesaki, On the equivalence among the components of decompositions of representations of involutive Banach algebras and the associated diagonal algebras, Tôhoku Math. J., 15 (1963), 365-393.

48. Covariant representations of $C^{*}$-algebras and their locally compact automorphism groups, Acta. Math., 119 (1967), 273-303.

49. - Duality for crossed products and the structure of von Neumann algebras of type III, Acta. Math., 131 (1973), 249-310.

50. G. Zeller-Meier, Produits croisés d'une $C^{*}$-algèbre par un groupe d'automorphismes, J. Math. Pures et Appl., 47 (1968), 102-239.

Received April 9, 1980 and in revised form July 28, 1980.

Drexel University

Philadelphia, PA 19104 



\section{PACIFIC JOURNAL OF MATHEMATICS}

\section{EDITORS}

DONALD BABBITT (Managing Editor)

University of Galifornia

Los Angeles, California 90024

HUgo RossI

University of Utah

Salt Lake City, UT 84112

C. C. MOORE AND ANDREW OGG

University of California

Berkeley, CA 94720

\section{J. DUGUNDJI}

Department of Mathematics University of Southern California Los Angeles, California 90007

R. Finn and J. Milgram

Stanford University

Stanford, California 94305

\section{ASSOCIATE EDITORS}
R. ARENS
E. F. BECKENBACH
B. H. NeUManN
F. WOLF
K. YosHIDA

\section{SUPPORTING INSTITUTIONS}

UNIVERSITY OF ARIZONA

UNIVERSITY OF BRITISH COLUMBIA

CALIFORNIA INSTITUTE OF TECHNOLOGY

UNIVERSITY OF CALIFORNIA

MONTANA STATE UNIVERSITY

UNIVERSITY OF NEVADA, RENO

NEW MEXICO STATE UNIVERSITY

OREGON STATE UNIVERSITY
UNIVERSITY OF OREGON UNIVERSITY OF SOUTHERN CALIFONIA STANFORD UNIVERSITY UNIVERSITY OF HAWAII UNIVERSITY OF TOKYO UNIVERSITY OF UTAH WASHINGTON STATE UNIVERSITY UNIVERSITY OF WASHINGTON 


\section{Pacific Journal of Mathematics}

\section{Vol. 93, No. $1 \quad$ March, 1981}

Richard Arens, Reducing the order of a Lagrangian $\ldots \ldots \ldots \ldots \ldots \ldots \ldots$

Richard Arens, Manifestly dynamic forms in the Cartan-Hamilton treatment

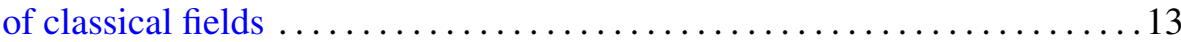

Jimmy T. Arnold, Power series rings over discrete valuation rings $\ldots \ldots \ldots 31$

Charles A. Asmuth and Joe Repka, Supercuspidal components of the

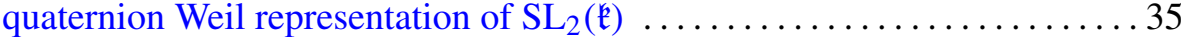

Luis A. Caffarelli and Avner Friedman, Sequential testing of several simple hypotheses for a diffusion process and the corresponding free boundary problem ................................. 49

William B. Jacob, Fans, real valuations, and hereditarily-Pythagorean fields .............................................. 95

W. J. Kim, Asymptotic properties of nonoscillatory solutions of higher order

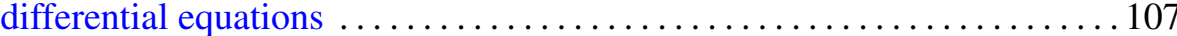

Wayne Steven Lewis, Embeddings of the pseudo-arc in $E^{2} \ldots \ldots \ldots \ldots \ldots 115$

Daniel Alan Marcus, Closed factors of normal Z-semimodules . ......... 121

Mitsuru Nakai and Leo Sario, Harmonic functionals on open Riemann surfaces ............................................. 147

John Currie Quigg, Jr., On the irreducibility of an induced

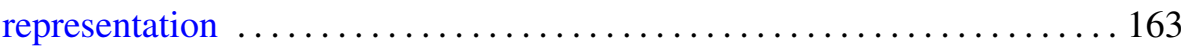

John Henry Reinoehl, Lie algebras and Hopf algebras 181

Joe Repka, Base change for tempered irreducible representations of $\mathrm{GL}(n, \mathbf{R})$

Peter John Rowley, Solubility of finite groups admitting a fixed-point-free automorphism of order $r s t$. I . . . . . . . . . . . . . . . . . . 201

Alan C. Woods, The asymmetric product of three homogeneous linear forms 